\title{
“Quill” Writing with Ultrashort Light Pulses in Transparent Optical Materials
}

\author{
Peter G. Kazansky, Weijia Yang and Erica Bricchi \\ Optoelectronics Research Centre, University of Southampton, SO17 1BJ, United Kingdom \\ James Bovatsek and Alan Arai \\ Applications Research Laboratory, IMRA America, Inc, Fremont, CA 94538, USA
}

\begin{abstract}
Writing in silica glass in opposite directions can be different. The phenomenon resembles quill writing and is interpreted in terms of anisotropic trapping of electron plasma by a tilted front of the ultrashort laser pulse.
\end{abstract}

Recently, direct writing in transparent materials by intense ultrashort light pulses has attracted considerable interest due to new applications and phenomena ranging from 3D optical waveguides [1], micro-explosions [2] and laser nano-surgery to photonic crystals and 3D self-organized sub-wavelength structures [3, 4]. It is well recognized that reversing the writing direction should not affect material processing and associated modifications. Here we report the observation of a new phenomenon in direct writing and ultrafast laser processing in transparent optical materials, in particular silica glass, manifested as a change in material modification by reversing the writing direction. The effect resembles writing with a quill pen and is interpreted in terms of new physical effect anisotropic trapping of the electron plasma by a tilted front of the ultrashort laser pulse.

The laser radiation, in a Gaussian mode, produced by a regeneratively amplified mode-locked Ti:Sapphire laser (150-fs pulse duration, $250 \mathrm{kHz})$, operating at $800 \mathrm{~nm}$, was focused via a $50 \mathrm{X}(\mathrm{NA}=0.55)$ objective into the sample. The beam spot size at the focus was $1.5 \mu \mathrm{m}$.

A series of 100 lines, with $7-\mu \mathrm{m}$ spacing, were directly written by scanning in alternating directions towards the edge of the sample at a depth of $0.5 \mathrm{~mm}$ below the front surface. The writing speed was $200 \mu \mathrm{m} / \mathrm{s}$ and each line was written with only one pass, in one direction, of the laser, with the polarization directed perpendicular to the line and pulse energy of $0.9 \mu \mathrm{J}$.

After writing, the structures were sidepolished and imaged with a Scanning Electron Microscope (SEM). The SEM images revealed

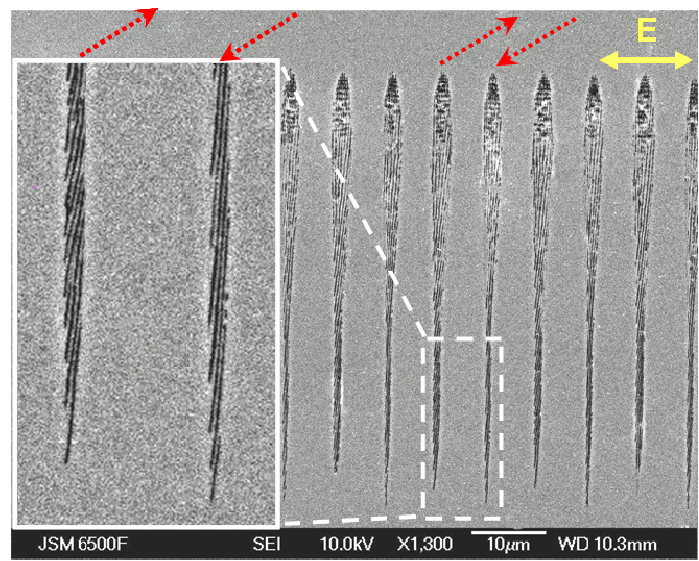

Figure 1 SEM images of cross sections of the structures in glass along light propagation written in opposite directions.

small variations of the length of the tracks and

of a tilt of the periodic structures written in the forward and reverse directions (Fig. 1). The periodic planar nanostructures are aligned along the direction of the writing laser polarization and are responsible for form birefringence of the irradiated regions.

In another experiment we wrote a series of lines using an IMRA-FCPA $\mu$ Jewel D-400 amplified ytterbium fiber laser system, operating at $1045 \mathrm{~nm}$, with pulse duration $<500 \mathrm{fs}$ and repetition rates ranging from $100 \mathrm{kHz}$ to $1 \mathrm{MHz}$. The high stability of the FCPA laser system is crucial for systematic studies [5]. The polarization of the laser was aligned perpendicular to the writing direction. The lines were written in alternating directions from forward to reverse and using different pulse energies ranging from 0.2 to $1.8 \mu \mathrm{J}$. After writing, microscope images were captured using both crossedpolarized and Nomarski-DIC illumination. Lines written in both directions at low energies were the same. However, with an increase in energy we observed the appearance of directional dependence in the written lines, which was strongest at about $0.8-0.9 \mu \mathrm{J}$ (Fig. 2a). The directional dependence is more clearly seen in the birefringence of the lines. This dependence can also be observed in the morphology 
(texture) of lines written in opposite directions, with a line written in one direction being rougher than a line written in the reversed direction (Fig. 2a).

An intriguing result is the observation of different textures in the processed material for laser polarizations perpendicular and parallel to the movement of the sample in one direction and the same textures for two polarizations when writing in the opposite direction (Fig. 2a).

(a)

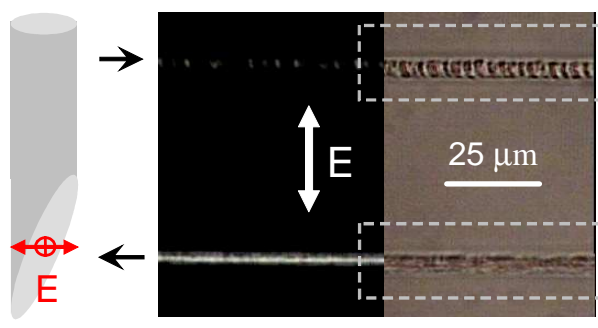

\section{Same}

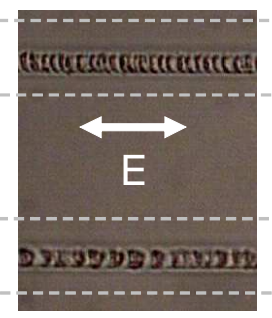

Different

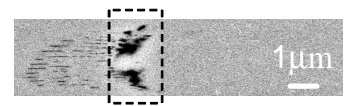

$\lambda / \mathrm{n}$

(b)

Figure 2 (a) Optical microscope images of the lines written with orthogonal polarizations with $500 \mathrm{kHz}$ repetition rate, writing speed $250 \mu \mathrm{m} / \mathrm{s}$ and pulse energy $0.9 \mu \mathrm{J}$. The difference in texture for two polarizations is observed only for one writing direction. The tilted front of the pulse along writing direction is shown. (b) SEM images of cross sections of lines written with polarization perpendicular to writing direction are also shown. The region of collateral damage is marked with black dashed line.

The SEM images of the cross-sections of the lines, along the light propagation, revealed a different texture in the lines written in opposite directions (Fig. 2b). Remarkably, the nanograting of about 300 $\mathrm{nm}$ period, which is responsible for the form birefringence of irradiated regions, can be seen only in the initial part of cross sections of lines written in one of two directions. This small area is followed by one with a collateral damage due to thermal effect, which correlates with a weak birefringence of these lines. It is also observed, that in almost entire cross sections of the lines, written in opposite direction, there is the nanograting along the direction of light polarization with the period of about $250 \mathrm{~nm}$ together with the additional periodicity, along the direction of light propagation, of about $720 \mathrm{~nm}$, which is of the wavelength of light $(\lambda / \mathrm{n}, \lambda=1045 \mathrm{~nm}, \mathrm{n}=1.45)$ (Fig. 2b). These lines demonstrate no evidence of the collateral thermal damage and much stronger birefringence (Fig. 2a).

The puzzle of the writing direction anisotropy is explained by the anisotropy of the frequency distribution (frequency chirp) and corresponding tilt in the intensity distribution in the front of the pulse [6]. It is known, that in the presence of intensity gradients, the charges (e.g. electrons) experience the pondermotive force (light pressure), which expels the electrons from the region of high intensity [7]. By moving the beam, the pondermotive force in the front of the pulse will trap and displace the electrons along the direction of movement of the beam and only in one direction corresponding to the tilt in the intensity distribution (we refer to this phenomenon as the "quill effect"). Further support of the proposed mechanism is the evidence of different textures of modified material for writing with light polarizations parallel and perpendicular to the movement in one of writing directions (Fig. 2a). This observation is explained by the difference in boundary conditions for two orthogonal polarizations at the interface of the tilted pulse front along the writing direction. In conclusion, it is remarkable that a laser beam, one of the most modern writing tools, could be used for calligraphic inscription similar to writing with a quill pen, which is based on the anisotropy of a quill's tip shape.

\section{References}

[1] K. M. Davis, K. Miura, N. Sugimoto, and K. Hirao, Opt. Lett. 21, 1729 (1996).

[2] E. N. Glezer and E. Mazur, Appl. Phys. Lett. 71, 882 (1997).

[3] Y. Shimotsuma, P. G. Kazansky, J. Qiu, and K. Hirao, Phys. Rev. Lett. 91, 247405 (2003).

[4] C. Hnatovsky, R. S. Taylor, E. Simova, V. R. Bhardwaj, D. M. Rayner, and P. B. Corkum, Opt. Lett. 30, 1867(2005).

[5] W. Yang, E. Bricchi, P. Kazansky, J. Bovatsek, and A. Arai, Optics Express 11, 10117 (2006).

[6] S. Akturk, M. Kimmel, P. O'Shea, and R. Trebino, Optics Express 11, 491 (2003).

[7] W. L. Kruer, The Physics of Laser Plasma Interactions, Addison-Wesley (1988). 